\title{
ACUTE MYOCARDIAL INFARCTION
}

\author{
R. W. D. Turner, O.B.E., M.A., M.D., F.R.C.P., F.R.C.P.E. \\ Senior Lecturer in Medicine, University of Edinburgh. \\ Physician, Physician-in-Charge of the Cardiac Department, \\ Western General Hospital, Edinburgh.
}

\section{Clinical Features and Diagnosis}

The usual history and clinical picture of acute myoracardial infarction is familiar and does not warrant detailed description here. Most often cardiac pain is retrosternal in situation and constricting in character with or without radiation to shoulders, arms, throat or jaws but there are many variations. The pain usually comes on suddenly and then increases. It may be very severe indeed and as bad as any known to man. Occasionally, despite unequivocal evidence of infarction, there may be no complaint of pain at all. In the latter instance pain may be overshadowed by some more impressive manifestation such a pulmonary œdema or syncope or there may be retrograde amnesia, but painless infarction is an established entity. Even when pain is not severe it is usually described as being very unpleasant. In some cases there is only a sense of oppression in the chest but whilst describing the symptoms, the patient may place his hand over the upper thorax and perhaps close the fist, thus depicting the diagnosis better than words. Rarely pain is felt only over the left paæcordial region or at the apex of the heart or only peripherally in jaws or wrists.

Occasionally the attack precipitates an arrhythmia which may be the dominant clinical finding. Sometimes infarction is heralded by the sudden onset or excerbation of angina of effort and the physician is surprised to find electrocardiographic evidence of old or recent infarction.

In a severe attack the principle feature may be shock or, if left ventricular failure has occurred, pulmonary œdema.

Sometimes at the bedside, objective confirmatory evidence that the patient has had_a heart attack may be found by noting that the jugular venous pressure is raised or the systemic pressure is low or that a triple rhythm can be heard.

In most cases an electrocardiogram (ECG), when first recorded, will show the pattern of infarction or of acute ischemia, but in some cases changes will only be evident in serial records and occasionally only if special leads are taken, such as those high up on the left side of the chest. A full series of precordial leads may be persistently negative in cases of infarction but this is uncommon and in a clinically doubtful case, calls for consideration of other possible causes for pain.

It is, however, important to remember that cardiac pain may actually be due to a dissecting aneurysm of the aorta obstructing the coronary arteries, in which case anticoagulants will be contraindicated, or to massive pulmonary embolism causing a fall in cardiac output and acute coronary insufficiency, in which case anticoagulants may be given or, under particularly favourable circumstances, surgical treatment should be considered. Accuracy in diagnosis is therefore important.

The following day there may be fever, pericardial friction, leucocytosis, a raised sedimentation rate or an increase in the serum glutamic transaminase level and urobilinogen may appear in the urine, any of which help in confirming a suspected diagnosis.

Breathlessness, sweating or vomiting may occur and anxiety is usual.

Vomiting on the way to hospital or shortly after admission is frequently due to a previous injection of morphine but, as discussed below, this could usually be prevented.

\section{Fever}

Fever occurs in most cases of severe infarction and probably arises from the absorption of necrotic tissue. The temperature usually rises within 24 hours of the onset, rarely exceeds $102^{\circ} \mathrm{F}$., is at its height for $48-72$ hours and then subsides. Persistent fever suggests some complication or an error in diagnosis. There is no indication for special treatment. It has been claimed that the prognosis is worse in those with high fever but undoubtedly exceptions occur and there is no close correlation between fever and prognosis. The temperature may be 
subnormal if shock is present despite severe infarction.

\section{Leucocytosis}

A mild leucocytosis with increase in the proportion of polymorphs, occurs in many cases of infarction. Usually it develops within 48 hours, is maximal about the third day and subsides by the end of the first week. The total count is not often above 15,000 but may be very high and in general this reflects the severity of infarction.

\section{Sedimentation Rate}

In general the sedimentation rate reflects the degree of myocardial necrosis and a subsequent fall runs parallel with healing.

\section{Electrocardiography}

Whenever possible, electrocardiography should form part of the routine assessment. This is not of course because an accurate diagnosis is not often possible without it but because in its absence the examination is incomplete. The physician may remain in ignorance of useful information and some avoidable errors in diagnosis will be made.

In the differentiation of equivocal cases, positive ECG changes may clinch the diagnosis and negative findings may give added support in reassuring the patient as to the absence of evidence for heart disease. It must be emphasized that errors in diagnosis are frequently made.

As always ECG changes must be interpreted in the light of the clinical information. In particular it is very helpful to know if there has been a history of previous infarction, if severe hypertension is present or if the patient is on digitalis. Difficulties in interpretation may arise owing to previous infarction, multiple areas of infarction, bundle branch block, ventricular hypertrophy or ventricular tachycardia and changes may be confused with those due to pulmonary embolism or pericarditis.

In the majority of patients with acute myocardial infarction there will be unequivocal evidence of necrosis or ischaemia in the first ECG to be recorded after the onset. In some instances there may be no abnormality although diagnostic changes appear if serial records are taken. However infarction may undoubtedly occur without significant ECG changes even in serial records and it is particularly in this group that the estimation of transaminase is of value.

In the days following acute infarction many patients experience pain arising in the chest wall which may be difficult to distinguish from further ischaemia and in such instances als electrocardiography is of value.

\section{Serum Transaminase}

The enzyme glutamic oxalo-acetic tran $\overrightarrow{\mathrm{s}}$ aminase (GOT) is widely distributed in manty tissues including the heart and is liberated into the blood stream as the result of injuzy or death of metabolically active cells. Pa. ticularly high titres are found after myocardia infarction and this test is therefore of valưe when other causes may reasonably be exclude $\bar{\phi}$.

The normal level of GOT in the serum (SGOT) ranges from 5 to 40 units. Readings of 40-50 are borderline and those above definitely abnormal. Fortunately the level not influenced by meals, anticoagulants steroids.

Following acute myocardial infarction th level rises after about 6 hours, usually reaches 8 peak between 24 and 36 hours and gradualfy returns to normal after 3 to 7 days, although occasionally longer. It is therefore important that timing should be taken into consideration when deciding on the significance of the laboratory report. Serial readings are oftem required. The enzyme is stable up to 4 dợys, or longer if kept cool. Haemolysis must avoided in collecting the specimen.

The estimation of SGOT is also useful whe a patient already under treatment for my cardial infarction develops a fresh attack pain or some other manifestation compatible with extension of the infarct. It is of course in such cases as this that serial estimations are of greatest value since there will be a base line to which changes can be referred. The test is also of value when the ECG is equivoca or obscured by previous infarction, ventriculä hypertrophy or bundle block and in distinguisla ing between acute coronary insufficiency and actual myocardial infarction.

Very high levels ( $>500$ units) probab\& signify associated hepatic necrosis secondary to the fall in blood pressure. It is noteworth. that shock from any cause may be accompang ied by high levels .

\section{Acute Coronary Insufficiency}

In some cases cardiac pain is prolonged bu yet there is no evidence of myocardial infarce tion. This condition is sometimes referred to as "Acute Coronary Insufficiency" on the as" sumption that there is a disparity between blood supply and myocardial needs withou actual necrosis. In such cases the venous pressure is not raised, there is no triple rhythn 
the picture of shock or pulmonary oedema does not develop nor is there subsequent pericarditis, leucocytosis, fever, a raised sedimentation rate or increase in serum transaminase.

Sometimes there is a spontaneous onset of angina without obvious precipitating cause and this has been referred to as "impending infarction".

Although occasional instances of coronary disease localised to one small vessel occur, it is best to assume that the condition is usually widespread, that there is progressive coronary arterial disease and that the sudden onset of symptoms is due to thrombosis, subintimal hæmorrhage or protrusion of atheromatous material, with or without actual occlusion of the lumen.

In my experience there are some patients with clinical features very similar to those described above as "acute coronary insufficiency" but with minor evidence of myocardial necrosis in the form of slight fever or leucocytosis, a mildly raised sedimentation rate or SGOT level and electrocardiographically there may be minor abnormalities barely distinguishable from normal but definite changes in serial records.

Clinically and pathologically there is a continuous spectrum and in the early stages at least, it is unwise to modify treatment on the assumption that there is a different underlying basis between cases. In fact, usually there is almost certainly pathological evidence of myocardial necrosis in some degree, either localised or focal and diffuse.

Certainly in the individual patient it is a dangerous assumption to conclude that acute coronary insufficiency is a relatively benign disorder. It is impossible to forecast the outcome and sudden death may occur, so that in the early days rest in bed with anticoagulant treatment is the wisest course. However, in view of increasing evidence as to the lack If value of anticoagulants in the relatively good risk cases, this initial programme of treatment may be modified and it is my own practice, having started anticoagulants in all patients, to reconsider the likely clinical course after a few days.

\section{Differential Diagnosis of Myocardial Infarction}

There are a large number of causes for pain in the chest but few which should give any real difficulty in differentiation if sufficient care is taken over the history and physical examin- ation. The most important are pericarditis, pulmonary embolism, dissecting aneurysm of the aorta and upper abdominal disorders.

\section{Acute Pericarditis.}

Pericarditis, and in particular idiopathic "benign serous" pericarditis, may be responsible for severe pain in the chest and has often been mistaken for myocardial infarction. Radiation may occur to the neck, shoulder, left arm and back but rarely to the right arm or jaws.

A number of points are of assistance in differential diagnosis. In pericarditis the rub usually appears at the onset whereas it commonly appears on the second or third day following myocardial infarction. In pericarditis pain is more influenced by respiration and posture. Shock is more likely to indicate myocardial infarction but occasionally a profound fall in pressure may occur with pericarditis. Arrhythmias or defects of conduction suggest infarction.

Unfortunately ECG changes are nonspecific and variable. Characteristically, in acute pericarditis the ST segments are elevated with upward concavity in limb and precordial leads and there is subsequent inversion of the $T$ waves. There is no change in the $\mathrm{QRS}$ complex. In myocardial infarction there is often reciprocal ST displacement in standard leads 1-3 and in leads recorded over the right and left ventricles, with an upward convexity in the elevated segments, and often also changss in QRS. When pericarditis complicates myocardial infarction $Q$ waves are almost always present. Sometimes, however, there may be no change and similar patterns may occur in either condition. Even serial records do not always help.

Radiographically there may be no change or there may be apparent enlargement of the cardiac outline in either condition. There may be general impairment of pulsation in pericarditis and local impairment in infarction, local pulmonary infiltrations in acute pericarditis and pulmonary œdema in infarction.

Myocardial infarction may of course be associated with pericardial friction. It must also be remembered that bouts of pericarditis and pleurisy occasionally occur during convalescence from myocardial infarction as described below.

\section{Pulmonary Embolism.}

The distinction between myocardial infarction and massive pulmonary embolism is not 
always easy and the two conditions may be almost identical. Pulmonary embolism is most likely to occur after surgical operations, fractures, parturition, thrombophlebitis or the onset of atrial fibrillation. It may follow enforced rest in bed in the elderly and sometimes appears "out of the blue" even in an ambulant patient. It is a frequent complication of cardiac failure.

In pulmonary embolism, breathlessness is usually more prominent than pain, and even if pain is severe it is unlikely to radiate as in myocardial infarction. The venous pressure is often elevated at the onset and peripheral cyanosis may be intense. Shock or triple rhythm may occur in either condition. In pulmonary embolism dilatation of the pulmonary artery may be visible and palpable to the left of the sternum and the pulmonary second sound may be accentuated.

Electrocandiography may be of value in differentiation. In massive pulmonary embolism the signs are those of clockwise rotation and right ventricular dilatation, that is, right axis deviation, a shift of the transitional zone to the left in precordial leads, inverted $T$ waves over the right ventricle and incomplete right bundle branch block. ECG changes are usually more transient with pulmonary embolism than with myocardial ischæmia but may persist for a week or two. In less severe cases the ECG will be unaffected. Sometimes ECG changes develop from secondary myocardial ischæmia due to coronary insufficiency and in the early days only be evident in left ventricular leads.

Estimation of serum transaminase may be of assistance.

Pulmonary embolism may be followed by hæmoptysis, pleural pain or pleural effusion. Myocardial infarction, of course, is itself not infrequently complicated by pulmonary embolism and infarction.

\section{Dissecting Aneurysm of the Aorta.}

Dissecting aneurysm of the aorta may also be difficult to distinguish from myocardial infarction. Pain in the chest may be similar but tends to be more sudden in onset with an immediate, devastating, tearing quality and more widespread radiation to the back, neck, epigastrium or lower limbs. Bizarre manifestations may appear, such as paræsthesiæ, paralysis of a limb, loss of vision, dysphagia, abdominal pain, changes in the pulses or a difference in blood pressure in the two arms.

The development of an early diastolic murmur from aortic incompetence secondary to dilatation of the valve ring is a very character- istic sign. Jaundice may follow from absor结 tion of blood.

Radiography is not always practical b may show progressive dilatation of the aorte. Electrocardiography may be helpful in $n \vec{A}$ showing signs of myocardial infarction bự sometimes there are non-specific ischæmic changes and occasionally the pattern of my宂 cardial infarction is present from compressio of the coronary arteries by downward dissection of the aneurysm.

\section{Acute Abdominal Emergencies}

Perforation of a peptic ulcer, acute chole्षे cystitis and pancreatitis may all give rise to high epigastric or low retrosternal pain ang have been mistaken for myocardial infarction. but careful attention to the history and physicat examination, together with electrocardiographys should serve to differentiate these conditionsi which will not therefore be described here.

\section{TREATMENT OF MYOCARDIAL INFARCTION}

The immediate treatment of myocardia infarction consists in the alleviation of paing restlessness and anxiety and the provisionoof adequate nursing care. Later, treatment mp be required for shock, cardiac failure or ot complications. Subsequent arrangements must be made for convalescence and, in most cases a gradual return to normal activity.

Pain, restlessness and anxiety are all best relieved by an injection of morphine. If symps toms are severe this drug should be given intras venously. In any case, absorption is more rapid after intramuscular than subcutaneous injection. $15-30 \mathrm{mg}$. ( $\frac{1}{4}$ to $\frac{1}{2}$ grain) should be given and can be repeated, if necessary, withir? half an hour.

If symptoms are mild there is no indication for morphine and it is better withheld. A seda? tive, such as amytal, or a milder analgesic suclo as tab. codeine co., will suffice in such cases $>$

In view of the strong tendency for vomiting. to be induced by morphine, especially ins patients with myocardial infarction, and the obvious undesirability of this complication morphine should never be given alone. It mayew be combined with an injection of atropine, of hyoscine or an anti-histamine such as cyclizine

In many cases vomiting can be avoided bye giving a tablet of cyclizine 15 minutes before the injection.

\section{Removal to Hospital}

Treatment at home is difficult unless ade $\frac{?}{\mathbb{D}}$ 
quate nursing facilities are available. Relatives will naturally be concerned over many details and much is left to chance. It is a compromise on which patient, doctor and family must decide together, but I have no doubt that, on balance, after the first few hours, and except for those too ill to move, the acute stage is best managed in hospital.

In any case no patient should be "rushed to hospital" within the first twenty-four hours for the sake of anticoagulant treatment. This is the period of greatest danger to life and an uncomfortable ambulance journey with the psychological upset of sudden, and perhaps cold, nocturnal change to a strange environment can only be detrimental. If desired, it is perfectly safe to initiate anticoagulant treatment without laboratory control, as discussed below.

Treatment in hospital is relatively easy. There will be a standard regime modified to individual needs, but one with which the staff are familiar and into which most patients readily fit. Standard observations can be made at regular intervals and emergency measures are ready to hand by day and night.

\section{Rest in Bed}

Many physicians still adhere to the traditional six weeks' rule. In my opinion, this is unnecessarily long for most patients and a fixed period for cases of varying severity is illogical. By contrast, some physicians now get their patients up into a chair within a day or two of the onset. However, a period of three weeks in bed is probably advisable for most cases of average severity. In mild cases and for those who feel really well, sitting in a chair by the hospital bedside or in the same room, may soon be permitted for part of the day.

Initial rest, with resultant decrease in cardiac work, seems logical in order to allow time for a damaged myocardium to commence healing and to cover the period during which complications are most frequent.

For severely ill patients a longer period will, of course, be required. Too early ambulation may encourage the patient to take liberties. Too long in bed will engender anxiety and introspection.

Three weeks not only gives initial rest but impresses on the patient that there has been an illness of moderate but obviously not of great severity. He will appreciate that a return to work within a reasonable period is likely to be the reward of taking advice but that there may have to be some reduction in overall activities. He will realise that there may be some price to pay for health but this is not excessive and clearly his doctor is taking a reasonably optimistic view.

There can be no question that in the past restrictions were too great. There would seem now to be a danger of moving to the opposite extreme.

Oxygen

It is customary to give oxygen in all severe cases but the benefit derived is questionable. Certainly it is required if there is dyspnœea or pulmonary œdema and possibly it is of value for shock. I have not been impressed by the alleged property of alleviating severe pain. The administration of oxygen is usually uncomfortable and often engenders anxiety.

\section{Digitalis}

Digitalis should not be administered as a routine but only for the treatment of cardiac failure which does not respond to diuretics or to control the ventricular rate if there is atrial fibrillation.

Some physicians consider that digitalis is of value in the treatment of shock but there is little actual evidence for this opinion. In general it is best not to give a potentially toxic drug unless there is some special indication.

\section{Diuretics}

Diuretics are extremely valuable for the treatment of pulmonary odema from left ventricular failure. They should also be given for severe or persistent congestive failure. Mild peripheral œedema will usually clear of itself as the patient improves and it is best not to induce a diuresis which contributes to exhaustion and possibly to venous thrombosis.

\section{Quinidine}

Quinidine should not be given as a routine measure for the prevention of arrhythmias but only for the treatment of ventricular tachycardia, persistent flutter or atrial fibrillation or for multiple extrasystoles which may preceed ventricular tachycardia or fibrillation.

\section{Anticoagulants}

After twenty years the place of anticoagulants in the treatment of coronary artery disease remains unsettled, and except for those who have themselves carried out clinical trials, physicians can but read the same evidence and draw their own conclusions. With so much doubt and conflicting evidence, it would seem 
that at best there cannot be very much in it, either as regards the efficacy of short-term treatment in reducing the mortality, or of long-term treatment in influencing the ultimate prognosis. There is general agreement that anticoagulants are of some value in diminishing the incidence of thrombo-embolic complications. These may include extension of coronary thrombosis or fresh thrombosis, the laying down of clot on the endocardial surface of the heart, venous thrombosis and pulmonary embolism.

Some are of the opinion that the small gain from the prevention of venous thrombosis and pulmonary embolism is balanced by the risks of hæmorrhage. This, however, should not apply where good laboratory control is available. Despite criticism, the balance of opinion is in favour of anticoagulants, at least in the acute stage, for the relatively severe risk group and for impending myocardial infarction as judged by the sudden onset of angina or the syndrome of acute coronary insufficiency. Long term treatment and the treatment of the good risk group is more debatable. The general trend is to restrict their use to shorter periods in fewer patients.

All will agree that the prognosis of myocardial infarction is very different in the good and poor risk groups but it is impossible to tell in the first few days after the onset whether a patient will change from a good to a poor risk group. Consequently, our practice is to give anticoagulants to all patients at the onset of myocardial infarction but after a few days or weeks gradually to tail them off in those who are clearly in the good or fairly good risk group.

As regards long-term treatment, from the statistical point of view, it seems that young males are most likely to derive benefit, particularly during the first 6 to 12 months, but there is little evidence in favour of continuing treatment for a longer period than this.

\section{Administration}

Heparin interferes with the blood clotting sequence at several stages and has a rapid and transitory action because it acts solely on clotting substances already present in the circulating blood. It must be given by injection. A suitable dose is 100 to $150 \mathrm{mg}$. $(10,000$ to 15,000 international units) preferably by intravenous injection but if necessary intramuscularly. This should be followed by 7,500 units every six hours for 24-36 hours, by which time the oral anticoagulant, given simultaneousl, will be effective.

Phenylindanedione (Dindevan) is the ofal anticoagulant in most frequent use in this country. The initial dose is 150 to $300 \mathrm{~m}$. and the average daily maintenance dose usually 50-125 mg., to keep the prothrombin activi of the patient as near as possible to 15 to $\approx 0$ per cent of a normal individual tested eaQh day by the same method.

\section{Hamorrhage from Over-dosage}

Heparin. An antidote exists in the form $\overrightarrow{\overrightarrow{\vec{g}}}$ protamine sulphate, which should be given intravenously in the form of $1 \mathrm{ml}$. of a $1 \mathrm{per}$ cent solution to each 1,000 international units of heparin given in the last dose. A transfusion of fresh blood may also be required.

Phenylindanedione. Vitamin $\mathrm{K} 1$ by mouth in adequate doses will usually restore the prothrombin time to normal within eight hougs but, with severe hæmorrhage, it can be given by injection. It is a powerful drug and shoutd only be used with caution and only when specifically indicated. It may be used in smadi doses to reduce an unduly prolonged gr: thrombin time or when more than staght hæmorrhage has occurred. In the first instange 2.5 to $5 \mathrm{mg}$. may be sufficient, but 10 to mg. may be given of hæmorrhage is moge severe.

It should be emphasised that $10 \mathrm{mg}$. is $\stackrel{2}{-7}$ large dose and unless the situation is seriogs and it is not intended to continue with ant coagulants, it is better to give small repeat doses controlled by the prothrombin time there may be undue delay in regaining ant. coagulant control with, of course, risk of fresti complications of the type for which proph lactic treatment was instigated.

A transfusion of fresh blood will also be necessary in severe cases.

Contra-indications to treatment are recent bleeding from the gastro-intestinal tract, bloo dyscrasias, and severe hepatic or renal insuffos ciency and, as regards long-term therapy, inability of the patient to co-operate or lact of adequate laboratory control.

Risks of anticoagulant treatment. Hæmort hagic complications occur in about 2 to 3 per cent of patients and it is for this reason thot treatment requires co-operation by the patient and adequate laboratory control. Hæmatemesi melæna, hæmaturia or uterine bleeding may be the first indication of unsuspected underlying disease. 
Abrupt withdrawal of anticoagulants may be dangerous and the rebound phenomenon with thrombosis is a real risk, although in some cases it may be due to the restoration of the normal pattern of the natural history of the patient's disease.

\section{Care of the Bowels}

Only those who are severely ill need the imposition of the bed-pan. Apart from psychological effects it is undesirable from the physical point of view. The use of a bed-pan is often difficult and the effort required is greater than the use of a bed-side commode. Better still is the modern wheeled chair, with hollow seat which fits over the ordinary lavatory pan. If necessary, the patient can be lifted or helped on and off the chair. There is no hurry to encourage bowel movement for the first few days; thereafter light purgatives or an enema can be given as required.

\section{Subsequent Management}

Following acute myocardial infarction most patients settle down rapidly within a few days and thereafter make an uncomplicated recovery. Our practice in those of this group who are admitted to hospital, is to advise three weeks in bed, one week getting up and about the ward and four weeks at home gradually increasing activity under the supervision of the family doctor and then reporting for review before returning to work. This means no more than two months off work which minimises any potential economic handicap and the development of a cardiac neurosis, and gives ample time for readjustment.

During the second month, walking should be encouraged and gradually increased, short of pain, dyspnœa or undue fatigue, and initially walking upstairs can be limited and taken slowly. Exercise is not only of value in promoting a coronary collateral circulation but in improving effort tolerance. It should be explained that normal individuals who are out of training and take little exercise experience a striking increase in effort tolerance after a few days of walking or climbing during a holiday. Unquestionably, walking is the best form of exercise for the coronary patient. Competitive games should be abandoned.

Early to bed and early to rise means less hurry in the morning and more time for getting to work. The majority of patients can expect to return to their previous occupation or to modified work if this is unduly heavy. The principal problem is to obtain more suitable employment for the unskilled labourer but it is most important not to change a man's ocupation unless it is really unsuitable. For those with responsibility delegation of duties may be important but only in the severely disabled need premature retirement be considered. Social and civic responsibilities can frequently be curtailed without hardship and in fact many are glad of such a reasonable excuse. Emotional stress is unquestionably adverse and an understanding wife or husband can do much to prevent tension and other adverse factors.

Whether or not to advise giving up smoking is controversial but there can be no question that in some patients smoking aggravates angina and can produce quite striking changes in the electrocardiogram or ballistocardiogram which may be of clinical significance. Smoking has no beneficial effects other than satisfying a craving for this drug and has other disadvantages which are well known, in addition to ill effects on the cardiovascular system.

Alcohol may have a beneficial sedative effect but is not a coronary vasodilator. When taken in moderation it is probably the best of tranquillisers.

Over-eating should be avoided and also exercise after a meal. Reduction of weight in the obese is clearly an advantage. There is, as yet, no conclusive evidence that alterations in the quality or quantity of ingested fat effect human atherosclerosis but there is considerable presumptive evidence in its favour. If dieting is necessary for reduction of weight and in those with a high blood cholesterol it would be sensible to cut down the intake of animal fat and cook in vegetable oil. This can readily be done, without materially spoiling the pleasures of the table, if the habit of mind is formed of avoiding obviously fatty food, cutting visible fat off all meat, only taking lean bacon, pouring the top off the milk and getting accustomed to bread and scrape rather than thickly spread bread and butter. Such rules can be ignored on special occasions.

\section{COMPLICATIONS}

\section{Shock}

Varying statistics on the incidence and significance of cardiogenic shock following myocardial infarction, are largely explained by differences in the criteria used for diagnosis. Shock is not synonymous with hypotension but is a readily recognisable clinical state, of which a fall in blood pressure is but one manifestation. The other principle features are pallor, weak- 
ness, sweating, a rapid thready pulse and oliguria. Without these the diagnosis should not be made.

The previous blood pressure will influence the level at which shock appears.

Shock usually appears at the onset of myocardial infarction and in about 50 per cent of patients the episode is transient and not of serious significance although hypotension may reappear a few hours later in some cases.

Left ventricular failure may appear with the onset of shock and the simultaneous development of an arrhythmia may intensify hypotension.

The disturbed physiological mechanisms present have not been completely clarified. The blood volume is not significantly decreased and there is no close relationship with a low cardiac output.

Myocardial failure and peripheral circulatory collapse are the two factors which may presumably be responsible but the precise pathogenesis is obscure. Experimentally it has been shown that shock is not solely due to the amount of myocardial damage, nor can it be accounted for by tachycardia.

Simulation of coronary shock

Errors in diagnosis are sometimes made because the clinical picture assumed to be due to myocardial infarction with shock may be very similar to that occurring from other causes. These include: (1) cardiac tamponade; (2) rupture of a dissecting aneurysm of the aorta; (3) rapid ventricular tachycardia; (4) massive pulmonary embolism; (5) acute pulmonary œdema; (6) acute myocarditis; (7) fulminating bacterial infection; (8) cerebral hæmorrhage; (9) internal hæmorrhage; (10) anaphylaxis.

These conditions should therefore be kept in mind in any patient with atypical features for myocardial infarction.

\section{Treatment}

If initial treatment has been ineffective, pain and anxiety should be relieved by a further injection of morphine together with atropine, hyoscine or an anti-histamine as explained above.

Oxygen by mask or tent has been shown to raise the arterial oxygen tension and may help in such cases. The use of positive pressure apparatus is still controversial.

\section{Posture}

Recumbency or semi-recumbency is best unless there is pulmonary œdema, when the patient may have to be propped up. In general, the position of maximum comfort should used.

100 to $200 \mathrm{mg}$. hydrocortisone intre venously should be given first. If the blood pressure does not respond a vasoconstricter drug should be administered. Metaraminol ${ }_{\text {TS }}^{\circ}$ probably the best available preparation and has the advantage that it can be given intr\% venously, intramuscularly or subcutaneousl 5-10 mg. can be given by I.V. injection or 10-25 mg. by intramuscular or subcutaneous injeø. tion, repeated as required, or $200 \mathrm{mg}$. ca佣 be dissolved in $250 \mathrm{ml}$. 5 per cent glucose in water and given by intravenous drip at 9 rate required to control the blood pressure.

The vasoconstrictor drug formerly most fro quently used is noradrenaline. This has to given by constant intravenous drip, one infusio containing $4 \mathrm{mg}$. noradrenaline dissolved $\overrightarrow{\mathrm{w}}$ $500 \mathrm{ml}$. of glucose. It is given at a rate suffes cient to maintain the systolic blood pressute at about $100 \mathrm{~mm}$. Hg. A dose of $1 \mathrm{mg}$. of noradrenaline is equivalent therapeutically to $20 \mathrm{mg}$. of metaraminol.

An important point to stress is that if treat. ment has any chance of success it must be started as soon as possible. There is far form universal agreement as to the value of differeet methods of treatment and, as already indicatee the prognosis of severely shocked patients $\vec{s}$ bad.

\section{Pericarditis}

Pericarditis occurs in about 20 per cent patients with acute myocardial infarction and is usually recognised by the detection of peri cardial friction between the second and four days of the illness. Pain may be mistaken for a recurrence of myocardial ischæmia but tends to be aggravated by respiration, coughing posture. No special treatment is required and the prognosis is not adversely affected.

Fost Myocardial Infarction Febrile Syndrome

A much rarer type of pericarditis has come to be known as the "post myocardial infarction febrile syndrome". In these cases, friction $\overrightarrow{\sqrt{3}}$ heard between the second and tenth weeks of illness and persists for days or weeks. The condition has a characteristic tendency teg relapse. Pericardial effusion develops in mo than half the cases and there may be assoce ated pleuritis or pneumonitis.

Steroids frequently relieve pain and feve dramatically but there is a tendency to recure rence of symptoms when the dosage is reduced and because of this such treatment should n\& be given unless symptoms are severe. 
Pleural or pericardial effusion may be extensive and require paracentesis for the relief of symptoms. The exudates are often hæmorrhagic.

Anticoagulants should be avoided or cardiac tamponade may result.

\section{Post Myocardial Infarction Pain}

Following myocardial infarction, referred somatic pain in the chest wall is of frequent occurrence and not always easy to distinguish from a recurrence of true cardiac pain. It may develop within a few days or later and persist or recur for days, weeks or occasionally longer. Tender areas in the chest wall are often present and in such cases, pain may be relieved by the local injection of 1 per cent procaine or superficial "freezing" with an ethyl chloride spray.

Pain may be felt near the sternum or in the left pectoral region. It often comes at rest and may be aggravated by movement, posture or respiration, but the patient may be unable to distinguish its quality from the original cardiac pain so that there will be undesirable anxiety on this account.

The mechanism is presumably that of visceromotor reflex muscle spasm similar to that which occurs elsewhere, e.g., in the abdominal muscles with appendicitis. In the case of referred pain from the heart this peripheral somatic effect sometimes persists and becomes a very important secondary factor which is often perpetuated not so much by the underlying disease as by anxiety and unwarranted restriction on movement.

It is worth keeping in mind that once the pain of acute myocardial infarction has subsidised spontaneously or with treatment, persistent or recurrent pain in the chest wall is more likely to be of somatic than cardiac origin.

Due caution is of course required but if in doubtful cases there is no supporting evidence for further myocardial infarction by electrocardiography or the estimation of transaminase, strong reassurance is indicated and the general management of myocardial infarction and subsequent rehabilitation continued. Although thoroughly established, this syndrome is not sufficiently recognised, with consequent misinterpretation and mismanagement.

\section{Arrhythmias}

Cardiac arrhythmias following myocardial infarction are of serious prognostic importance and sometimes associated with sudden death. The incidence is higher in the older age groups.
Extrasystoles

Extrasystoles often develop. Supraventricular extrasystoles are less frequent and less important than ventricular extrasystoles. The physiological importance depends on the frequency with which they occur and the consequent effect on coronary blood flow and systemic blood pressure. More important is the fact that they may precede atrial fibrillation or ventricular tachycardia. Consequently, if they occur frequently quinidine should be given. If this fails, oral pronethalol $100 \mathrm{mg}$. 4-6 times a day cay be tried but watch kept for a fall in blood pressure. This drug is best for ventricular arrhythmias.

\section{Supraventricular tachycardia}

Supraventricular tachycardia is not a frequent occurrence but of serious significance. If the attack cannot be terminated by vagal stimulation, the patient should be digitalised. If necessary, vagal stimulation can then be tried again and is more likely to be effective. If the blood pressure is low, pressor amines should be given and may themselves convert the arrhythmias to sinus rhythm.

\section{Atrial fibrillation}

Mortality is little influenced by transient fibrillation but the persistent arrhythmia is of more serious prognostic importance. The ventricular rate should be controlled with digitalis and, if necesary, pressor agents should also be given. Anticoagulants should be continued in all such cases. It is best to delay the restoration of sinus rhythm with quinidine until after the acute phase of infarction is over unless the ventricular rate cannot be controlled.

\section{Atrial flutter}

The significance and management of atrial flutter is similar to that of atrial fibrillation.

\section{Ventricular tachycardia}

Ventricular tachycardia is responsible for many sudden deaths following myocardial infarction. Frequent ventricular extrasystoles should be taken as a warning sign of impending ventricular tachycardia and quinidine should be given prophylactically as mentioned above.

In urgent cases procainamide is superior to quinidine for treatment because it has a similar action, but can be used intravenously with more safety (100 mg. a minute up to $1 \mathrm{G})$. When ventricular tachycardia is due to digitalis intoxication potassium salts should be given.

If quinidine or procainamide fail pronethalol should be given in a dose of $100 \mathrm{mg}$. intravenously which can be repeated if necessary. 
This drug is often effective and can be continued by mouth until the danger period is over.

Decreased coronary flow may be responsible for the arrhythmia and increasing the systemic pressure with pressor amines may be successful in restoring sinus rhythm or in potentiating the effect of other drugs.

\section{Defects of Conduction}

Severe degrees of atrioventricular block are of serious prognostic significance. First and second degree blocks are usually asymptomatic but may procede to third degree block.

Complete A-V association carries a 50 per cent mortality and may be complicated by Adams-Stokes attacks.

Hydrocortisone (200 mg.) should be given intravenously in the first place and $100 \mathrm{mg}$. may be repeated every 4 to 6 hours as required. Sublingual or, if necessary, intravenous isopropylnoradrenaline (isoprenaline) may abolish the block. If these measures fail, adrenaline should be tried and, if the blood pressure is low, pressor amines should be given as supplementary treatment.

Another drug which has been used with success is molar sodium lactate.

\section{Cardiac Failure}

Cardiac failure after myocardial infarction may be in the form of pulmonary odema from left ventricular failure, or of the peripheral signs of increased venous pressure, cedema and congestion in the liver. Treatment should be on the usual lines with digitalis and diuretics.

\section{Embolism}

Pulmonary or systemic embolism is an important complication occurring usually in severe cases of myocardial infarction but has become less frequent following the introduction of anticoagulant drugs. Statistics compiled prior to 1948 suggest that thromboembolic complications occurred in at least 10 per cent of cases of myocardial infarction, accounted for about 10 per cent of deaths and contributed to more.

\section{Pulmonary Embolism}

Pulmonary embolism is frequently mistaken for further myocardial infarction. It may result from thrombosis in peripheral veins or occasionally, and especially if there is right-sided failure or atrial fibrillation, in the right atrium and less frequently from trans-septal infarction involving the right ventricular wall. It may occur in the first few days but more often after the first week and sometimes unexpectedly when the patient gets out of bed. It is for tho reason that anticoagulants should be continued over this period.

Systemic Embolism

Cerebral embolism with the resultant infar tion or softening of the brain and the develop ment of hemiplegia, aphasia or mental changes. is a not infrequent complication of myocardiat infarction. These effects may be difficult oo impossible to distinguish from similar mantै festations due to a fall in cardiac output with decreased blood flow in atheromatous cerebral or extracranial vessels. Emboli may lodge $\mathrm{i} \overrightarrow{\mathrm{b}}$ kidneys, spleen, mesenteric vessels or the limbs

\section{Rupture of the Interventricular Septum}

Involvement of the septum by myocardiat infarction is a frequent occurrence but peri foration is rare. This is a serious complicas tion and most patients die within a week or twe although occasional long survival has beeg reported. The physical signs are those of $\bar{a}$ systolic murmur and thrill maximal at the left sternal border. Successful surgical repair has been reported in a few cases and this. form of treatment may therefore reasonablyobse considered in patients who are deteriorat of if adequate facilities are available.

\section{Cardiac Aneurysm}

Cardiac aneurysm is an infrequent complêّ cation of myocardial infarction due to replaces ment fibrosis usually involving the anterior wa: of the left ventricle. Although sometimes sus pected by the palpation of a double cardiae impulse or an outward thrust during systoles the diagnosis is usually made by radiographyo There is an abrupt change of contour on the left heart border producing a shelf with reduce or paradoxical pulsation on radioscopy.

Electrocardiographic changes are not diago nostic but there is usually evidence of extensive infarction and in some cases persistent eleva tion of the ST segment in precordial leads.

Cardiac rupture is rare but incapacitating. symptoms may result from impairment of func tion produced by the expansile but non contractile aneurysm. Sometimes embolisn results from mural thrombosis.

The importance of recognising this condi? tion lies in the fact that in such cases refractor cardiac failure may respond dramatically tक surgical excision of the aneurysm.

\section{Frozen Shoulder}

Pain, stiffness and limitation of movemen at the shoulder joint is a well recogniseo 
complication of myocardial infarction but should be an infrequent one. In some cases swelling, stiffness and discolouration of the hands and fingers has followed.

The remarkable variation in the reported incidence of this syndrome is probably a reflection on the degree to which patients are immobilised in bed following myocardial infarction. In our Unit they have always been allowed to move about as freely as inclined and "frozen" shoulder has been a rare occurrence. The patient in pain or with shock will naturally lie still but we have always felt that immobility enforced by medical and nursing staff is liable to lead to complications and a degree of anxiety more adverse than any potential benefit. In uncomplicated cases, as referred to above, no patient has been kept in bed for more than three weeks, and is always encouraged to move about and be wheeled out to the lavatory in a special chair, on the assumption that the physical exertion and mental anguish of using a bedpan were adverse.

Although the condition may take months to improve, the syndrome, which is similar to that which may follow trauma, hemiplegia or cervical arthritis, is self-limiting and the long-term prognosis is good. Numerous forms of treatment have been recommended but the combination of analgesics and physiotherapy is usually sufficient. The local injection of hydrocortisone is probably of no benefit.

\section{Death}

The principle causes of death from myocardial infarction are:

1. Shock;

2. Cardiac failure;

3. Cardiac arrest;

4. Acute arrhythmias;

5. Thromboembolic complications;

6. Cardiac rupture.

Treatment may be effective in all except the last of these.

\section{Prognosis}

Coronary artery disease can rarely be diagnosed before symptoms are present and usually the onset of symptoms gives no indication of the extent of underlying disease. In fact, there is no medical condition in which the future is more uncertain and unpredictable. Analyses of large series do not help in the individual case but statistics, of course, do give overall guidance which is of value. The practitioner must instil confidence and optimism into the patient but cover all eventualities with the relatives. To the lay mind angina is associated with sudden death and this does in fact occur in some 10 per cent of cases. Sometimes, the increased frequency and severity of attacks have brought realisation of progression to all concerned but more often, when myocardial infarction occurs, although the practitioner will have had such eventualities in mind, it comes unexpectedly to the family.

Following myocardial infarction the prognosis is influenced adversely by a history of previous infarction or systemic hypertension; by the presence of cardiac enlargement; by evidence of extensive infarction as judged by prolonged pain, triple rhythm, shock, cardiac failure, extensive ECG changes or a highō level of SGOT; or by recurrence of pain? or by pulmonary or systemic embolism, an arrhythmia or defects of conduction.

Although the influence of long-term anticoagulant therapy in affecting life expectancy is uncertain, as discussed above, few would doubt the value of anticoagulants in severe cases, at least as regards their limiting effect on venous thrombosis and pulmonary embolism.

Many patients, of course, die before admission to hospital or before treatment can be instituted. Probably some 20 per cent who are admitted to hospital die within a month, but in the remainder the outlook is much better than used to be thought, especially in those who make a good recovery without complications or after effects. At least 75 per cent of those who leave hospital well will survive for five years and probably thereafter the mortality rate is not more than 5-10 per cent per annum. 